\title{
Laser radiation impact on a solid surface and formation of micro- and nanostructures
}

\author{
Dmitriy Kochuev*, Ruslan Chkalov, Valery Prokoshev, and Kirill Khorkov \\ Vladimir State University, 600000 Vladimir, Russia
}

\begin{abstract}
The work is devoted to the problem of micro- and nanostructuring of the surface of solids as a result of exposure to laser radiation. The method of forming periodic surface structures by means of femtosecond laser processing is presented using the example of solving the problem of titanium structuring.
\end{abstract}

\section{Introduction}

The formation of laser-induced periodic surface structures (LIPPS) is a unique phenomenon that can be observed on almost any material after irradiation with linearly polarized laser radiation, especially of femtosecond duration [1]. Such processing technology provides a simple method of nanostructuring and functionalization of the surfaces of materials to control their optical, mechanical or chemical properties [2]. The effect of femtosecond radiation on the metal surface leads to a decrease in the friction coefficient and a slowdown in wear of products, which emphasizes the huge potential of LIPPS technology in tribological applications. A distinctive feature of this method is the ability to process even refractory metals with high performance characteristics, the most widely used of which is titanium. In view of the above, an urgent task is to structure the titanium surface in order to form a titanium carbide coating and obtain an ordered relief of LIPPS structures [3].

\section{Experimental setup}

To process the surface of titanium samples, the following experimental scheme was used (Fig. 1): the sample was mounted on a stage, the vessel was sealed, the volume of the vessel was pumped out and filled with high-purity argon for 5 times, then the vessel was filled with n-hexane to the required level ( $1 \mathrm{~mm}$ above surface of the sample), the required pressure was established, after which the sample was scanned with a laser beam.

The pressure of the buffer gas in the vessel in the range from 0.0063 bar to 22 bar was used as a variable. The lower limit is due to the physical properties of n-hexane, which lead to intense boiling of the liquid, the upper limit of 22 bars is set by the technical characteristics of the gas fittings.

\footnotetext{
*Corresponding author:_b_@mail.ru
} 


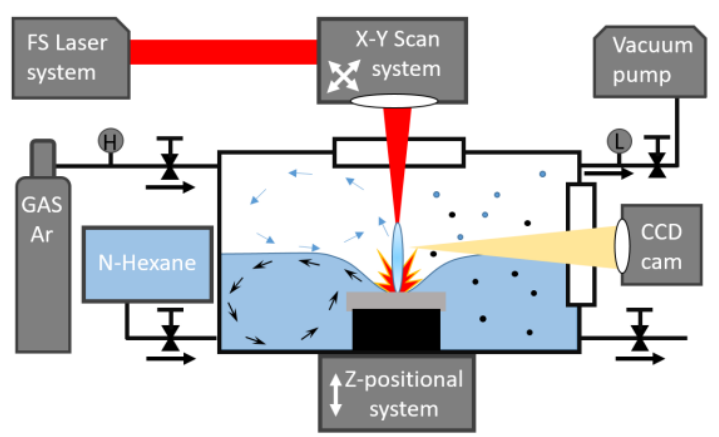

Fig. 1. Schematic representation of the surface treatment of a titanium sample in n-hexane.

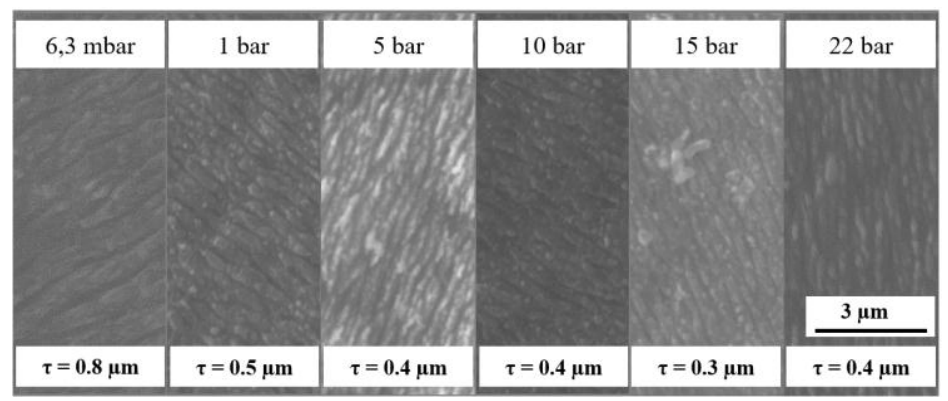

Fig. 2. The result of surface treatment of a titanium sample at various values of gas pressure, where $\tau$ is the period of LIPPS structures.

Figure 2 shows the results of treating the surface of a titanium sample with femtosecond laser radiation in accordance with given conditions. As can be seen from the illustration, a sequential increase in the pressure of the buffer gas leads to a number of patterns. In explicit form, there is a tendency to a decrease in the period of LIPPS structures, the dependence of the relief on the treatment mode. The boiling intensity of n-hexane is somewhat reduced, the color change of the liquid medium becomes less pronounced, the ablation dynamics of the titanium surface decreases.

\section{Conclusion}

Varying the conditions for LIPS processing, in particular the pressure of the external medium on the sample, leads to a significant change in the relief pattern of the structured surface. Thus, the selection of the optimal processing mode allows achieving unique parameters of nanostructuring, which entails a change in the operational properties of the product material.

\section{References}

1. Q. Litao, N. Kazuhiro, N. Yoshiharu, Opt. Let., 34 (2009).

2. A. Sabri, F. Fotis, T. Kunze et al., Materials, 12 (2019).

3. A. Voznesenskaya, D. Kochuev, A. Zhdanov et al., J. of Phys.: Conf. Ser., 1164 (2019). 